ФГБУ «Национальный медицинский исследовательский центр эндокринологии» Минздрава России, Москва, Россия

В настоящее время в мире основным диагностическим параметром ожирения считается величина индекса массы тела. У детей, с учетом динамически изменяющихся по мере взросления ребенка показателей роста и массы тела, принято пользоваться не абсолютными, а относительными значениями индекса массы тела - перцентилями или стандартными отклонениями. В лекции рассмотрены различные системы и способы оценки физического развития детей в мире и в России - отечественные, Р.Н. Дорохова и И.И. Бахраха, Всемирной организации здравоохранения (ВОЗ), Международной группы по изучению ожирения. Проведен сравнительный анализ существующих систем и обоснованность принятых в настоящее время Федеральных рекомендаций по диагностике ожирения у детей на основе рекомендаций ВО3.

КЛЮЧЕВЫЕ СЛОВА: ожирение у детей, индекс массы тела, критерии ожирения у детей

\title{
OBESITY IN CHILDREN AND ADOLESCENTS: DIAGNOSIS CRITERIA
}

\author{
(C) Olga V. Vasyukova
}

Endocrinology Research Centre, Moscow, Russia

Currently in the world the main diagnostic parameter for assessing obesity is the magnitude of body mass index. In children, taking into account the growth and body weight indicators that dynamically change as the child grows up, it is common to use not absolute, but relative values of body mass index - percentiles or standard deviations. The lecture examined various systems and methods for assessing the physical development of children in the world and in Russia - domestic ones, R.N. Dorokhova and I.I. Bakhraha, World Health Organization (WHO), International Group for the Study of Obesity. A comparative analysis of the existing systems and the validity of the currently adopted Federal recommendations on the diagnosis of obesity in children based on the recommendations of WHO has been carried out.

KEYWORDS: obesity in children, body mass index, criteria for obesity in children

Как и любое другое заболевание, ожирение у детей и подростков требует четких, доступных и удобных для использования в практическом здравоохранении критериев диагноза.

Казалось бы, что может быть проще - и так все очевидно при простом визуальном осмотре - тучный ребенок или с нормальной массой тела, что же здесь сложного.

Но вместе с тем, до настоящего времени в России и в мире не достигнуто единого мнения по критериям диагностики ожирения у детей. Отсутствие единых стандартов объясняет противоречия в оценке распространенности данного состояния даже в пределах одной популяции. Наряду с этим, существует клинически доказанная аксиома, что успешность борьбы с детским ожирением начинается с его раннего выявления на начальной стадии в юном возрасте, и такой подход, как показал опыт ряда европейских стран, позволяет успешно решать проблему, избегая выраженных форм в подростковом периоде.

В чем же сложность? Попробуем разобраться, опираясь на данные литературы и привычную практику, сложившуюся в нашей стране.

Для диагностики ожирения у детей и подростков необходимо определиться с тремя составляющими:

1. Критерий ожирения: им может быть масса тела (вес), индекс массы тела (ИМТ), окружность талии; толщина кожной складки, величина жировой массы, оцененная с помощью дополнительных визуализирующих методик (биоимпедансометрия, КТ; МРТ жировой ткани).

2. Контрольная выборка: национальные данные, популяционные исследования других стран, международные исследования.

3. Референсные значения исследуемого параметра ("cut offs"): например, 95 или 97 перцентиль кривой ИМТ, стандартные отклонения и др.

Антропометрические показатели составляют абсолютное "популярное большинство" среди всех имеющихся на сегодняшний день в мире методов оценки физического развития. В первую очередь это связано с тем, что изменения показателей длины и массы тела растущего организма являются одним из основных показателей состояния здоровья ребенка, его нутритивного статуса. В большинстве случаев именно отклонения от нормальных значений прибавок роста и массы тела являются первыми признаками заболеваний, обязывающими врача проводить углубленное обследование ребенка. Во вторых, популярность антропометрических методов объяснима их доступностью, дешевизной и простотой в применении.

Начиная с 60-х годов прошлого столетия, для оценки гармоничности физического развития ребенка использовали простое соотношение массы тела с длиной тела. В этот же период времени у взрослых для определения нормальной массы тела были популярны различные индексы: индекс Брока (используется при росте 155-170 см; нормальная мас- 
са тела при этом определяется как (рост [см] - 100) $\pm 10 \%) ;$ индекс Брейтмана (нормальная масса тела - это рост [см] • 0,7 - 50 кг), индекс Татоня (нормальная масса тела как рост $(100+($ рост - 100) / 20) и другие.

В 1972 году американский ученый Ансель Бенджамин Kис (Ancel Benjamin Keys) в своей статье на страницах журнала the Journal of Chronic Diseases предложил использовать для оценки избыточности веса показатель "индекс массы тела" (ИМТ), разработанный еще в 19-м веке бельгийским социологом и статистиком Адольфом Кетле (LambertAdolph-Jacques Quetelet) [1]:

ИМТ (индекс Quetele) = вес (кг) / рост $\left(\mathrm{M}^{2}\right)$.

С этого времени начинается активное применение ИМТ в клинической практике для оценки массы тела.

Однако определение "нормы ИМТ" у взрослых неоднократно изменялось. Так в США до 1998 года считался "нормальным" ИМТ до 27.8 кг/м². В это время, когда Соединённые Штаты были на пике своего рода эпидемии сердечно-сосудистых заболеваний, Американская ассоциация по изучению сердца (The American Heart Association) включает ожирение как один из серьезных факторов риска сердечно-сосудистых катастроф, и по рекомендации ВОЗ 1997 году стандарты ИМТ были изменены:"норма" ИМТ стала“заканчиваться" на 25 кг/м². Из-за этого изменения стандартов около 29 миллионов ранее "здоровых" американцев стали считаться имеющими избыточный вес и ожирение.

Кроме этого, предложенные ВОЗ в 1997 году нормативы по оценке ИМТ, активно использующиеся у взрослых и в настоящее время, не учитывают пол, возраст и этническую принадлежность человека. Хотя исследования многих стран показывают, что ИМТ выше у людей среднего возраста, чем у молодых и пожилых, у мужчин по сравнению сженщинами, но ниже у азиатов.

Что же в педиатрической популяции?

В 1979 году британский ученый Тим Коул (Tim J. Cole), проведя исследование по динамической оценке росто-весовых показателей у 4631 детей из 5 стран, показал, что изменения массы тела и роста у детей в различные возрастные периоды (младенчество, раннее детство, пубертатный период) неравномерны, чем обосновал необходимость дополнительной оценки ИМТ с учетом возраста ребенка [2].

В 1982-1984 гг французская исследовательница Мария Франсуаза Ролланд-Качера (Marie Françoise Rolland-Cachera) составила первые центильные таблицы ИМТ (на основе национальной выборки) и ввела понятие "времени перестройки жировой ткани" («adiposity rebound») - время превалирования прибавки массы над прибавкой роста.

В дальнейшем в работах многих исследователей было показано, что значение ИМТ у детей меняется с развитием ребенка: от высокого в первый год жизни, сниженного в период раннего детства (2-5 лет) и увеличивающегося в период полового развития, что в целом отражает динамику жировой ткани. Так, на 1 кг массы плода жировая ткань составляет 2,5\%, при рождении ребенка - 12\%, а к 6 месяцам достигает $25 \%$. После этого соотношение жировой ткани к мышечной снижается до начала полового созревания. У девочек после 8-9 лет процент жировой массы быстро увеличивается вплоть до 17 лет. У мальчиков в период полового созревания жировая масса нарастает медленно и начинает снижаться после 13 лет.

Точкой отсчета для "adiposity rebound" является возраст минимального значения ИМТ, который в европейской по- пуляции наблюдается в 6,0-6,5 лет. Считается, что данный показатель является критическим периодом для развития ожирения впоследствии. Исследования М.Ф. Ролланд-Качера с соавт. показали, что дети с "ранним" (<5,5 лет) "adiposity rebound" имели статистически значимо более высокий ИМТ в возрасте 21 года по сравнению со сверстниками со "средним" (6-6,5 лет) и “поздним" "adiposity rebound". В дальнейшем было показано, что раннее начало «adiposity rebound» является фактором риска развития ожирения во взрослом возрасте, вне зависимости от наличия или отсутствия ожирения у родителей $[3,4]$.

Таким образом, с 1990-х годов прошлого столетия для оценки избыточной массы тела и ожирения у детей большинство стран мира начинают использовать центильные таблицы оценки ИТМ у детей с учетом пола и возраста. Т.е. показатели роста и веса конкретного ребенка сравнивают со статистическими значениями соответствующей возрастной группы. Центильные шкалы представляют собой описание частотных долей распределения диапазона варьирования признаков, абсолютно независимое от математического распределения. Соответственно эти шкалы универсальны, удобны при массовых обследованиях детей, для выделения групп с"пограничными" значениями и возможными патологическими отклонениями признаков. В Европе чаще используют шкалу Стюарт, в которой предусмотрено выделение границ 3, 10, 25, 50, 75, 90 центилей распределения, в США чаще применяют крайние частотные точки 5 и 95 центилей. При этом "нормальные значения" ИМТ, основанные на результатах популяционных исследований, значительно разнятся между странами. Кроме этого, со временем изменяются референсные значения ИМТ даже в пределах одной популяции: так, в США первоначально диагноз ожирения у детей и подростков устанавливался при значении ИМТ, превышающем 85 перцентиль, а в дальнейшем - при ИМТ более 95 перцентили [5].

В нашей стране до недавнего времени оценка физического развития детей проводилась путем определения соматотипа по методу Ратмира Николаевича Дорохова и Ильи Исаковича Бахраха в модификации Игоря Михайловича Воронцова $[6,7,8]$.

Впервые советские ученые Р.Н. Дорохов и И.И. Бахрах, используя популяционные данные, в 1975 году предложили определение соматотипов на основании величин сигмальных отклонений длины, массы тела и окружности грудной клетки от средних значений. Принадлежность ребенка к одному из трех соматотипов (микросоматический, мезосоматический, макросоматический) оценивали по сумме номеров центильных интервалов (зон), полученных для длины, массы тела, окружности грудной клетки, с учетом возраста и пола. В 1985 году эти интервалы названы И.М. Воронцовым «коридорами». При сумме баллов до 10 ребенка относили к микросоматотипу (данные обследуемого ребенка находятся в пределах 3-10 центиля), при сумме от 11 до 15 баллов к мезосоматотипу (все антропометрические показатели находятся в пределах 25-75 центиля), а от 16 баллов - макросоматотипу (полученные результаты соответствуют 90-97 центилю).

По мнению И.М. Воронцова, соматотип применительно к ребенку означает не столько тип телосложения, сколько темповую характеристику роста: микросоматотип - замедленный, макросоматотип - ускоренный, мезосоматотип - 
средний темп роста. При дисгармоничном развитии соматотип не определяется.

К несомненным преимуществам предложенной оценки физического развития детей относится возможность использования с первого месяца жизни до 17 лет (как определялся период детства в советское время), ее системность и многогранность, которая в сравнении с простым определением перцентилей ИМТ у детей позволила бы избежать существующих в современности как "недооценки" (у высоких) так и "переоценки" (у атлетов) значений ИМТ. Среди недостатков - отсутствие новых популяционных измерений (разработана на данных послевоенной популяции).

В настоящее время в мире наиболее популярными являются три системы оценки физического развития ребенка и ИМТ - американские, разработанные Центрами по контролю и профилактике заболеваний США (U. S. Centers for Disease Control and Prevention - CDC), критерии Международной группы по изучению ожирения (International Obesity Task Force - IOTF) и нормативы Всемирной организации здравоохранения (ВОЗ). Каждая из них имеет свои таблицы нормы роста, веса и ИМТ, а также критерии избыточной массы тела и ожирения у детей и подростков.

Американская система CDC разработана на основе национальной выборки и включает данные пяти репрезентативных кросс-секционных исследований, проведенных в США с 1963 по 1994 г. Согласно таблицам, значения ИМТ выше и равные 85-й перцентили ИМТ оцениваются как избыточная масса тела, выше и равные 95-й перцентили - как ожирение $[9,10]$.

Несомненным преимуществом данной системы является разнообразие и охват выборки, к недостаткам следует отнести возраст оценки от 2 лет и невозможность индивидуальной оценки величины ИМТ в динамике.

Критерии IOTF разработаны на основе шести больших кросс-секционных национальных исследований, проведенных в Бразилии, Великобритании, Гонконге, Нидерландах, Сингапуре и США в период с 1963 по 1993 г. среди мужчин и женщин старше 18 лет. В результате этих исследований были определены значения ИМТ, соответствующие избыточной массе тела (ИМТ $=25$ кг/м²) и ожирению (ИМТ равный и превышающий 30 кг/м²) у взрослых. В дальнейшем математически были рассчитаны нормы ИМТ для детей и подростков от 2 до 18 лет. К ограничениям данной системы также относится возраст оценки от 2 лет и отсутствие возможности объективной оценки изменения ИМТ в динамике [11].

В основе норм роста и веса, предложенных ВОЗ - проспективное наблюдение в течение первых 24 месяцев жизни с последующим перекрестным обследованием через 18-71 месяц 8440 здоровых младенцев из 6 стран (Бразилия, Гана, Индия, Норвегия, Оман и США). Все дети воспитывались в среде, способствующей физиологическому росту грудное вскармливание, сбалансированные рационы питания, профилактические прививки, отказ матери от вредных привычек и т.д. При сравнении с ранее имевшимися послевоенными данными оказалось, что дети, растущие в здоровых условиях, выше, худее и умнее. На основе этих исследований разработаны нормы роста и веса детей от 0 до 5 лет. Дальнейший анализ полученных данных с привлечением математического моделирования и выборки США (репрезентативное кросс-секционное исследование среди детей различных этнических групп) позволил разработать нормы роста и веса для детей от 5 до 19 лет включительно [12].
Главным преимуществом данной системы оценки является методологический подход: оценка как "должен" расти здоровый ребенок, а не как он растет в современном обществе, общая "тучность" которого увеличивается каждое десятилетие. Кроме этого, нормативы ВОЗ позволяют оценивать антрометрические показатели у детей от 0 до 19 лет, унифицированы и удобны для индивидуальной оценки в динамике ("дельта" SDS ИMT).

BO3 пользуется стандартными отклонениями ИМТ (SDS ИМТ) от 0 до 3. Согласно рекомендациям ВO3, ожирение у детей и подростков 5 лет и старше следует определять как $+2,0$ SDS ИMT, а избыточную массу тела от +1,0 до +2,0 SDS ИMT.

На сайте ВОЗ представлены нормативные значения роста и веса для детей в виде таблиц и кривых как для возрастной группы от 0 до 5-ти лет (http://who.int/childgrowth/ standards/ru/) так и для детей 5-19 лет (http://who.int/ growthref/who2007_bmi_for_age/en/index.html).

На сайте ВОЗ также доступны для скачивания и пользования компьютерные программы для оценки физического развития - Anthro (для детей в возрасте 2-5 лет) и AnthroPlus (для детей в возрасте 5-18 лет).

Нормативы ВОЗ на сегодняшний день применяются в 38 странах Европейского региона.

Единой точки зрения на выбор унифицированной общей системы оценки ожирения у детей в мире нет, хотя абсолютное большинство стран использует в основе определения нормы массы тела величину ИМТ.

На основе существующих систем оценки многие государства разработали собственные стандарты, учитывающие национальные особенности.

При сравнении сопоставимости существующих норм веса у детей данные варьируют[13, 14].

Так, национальные критерии определения ожирения у детей во Франции сопоставимы с нормами ВОЗ и значительно ниже значений IOTF, тогда как определение избыточной массы тела примерно одинаковое по исследуемым системам оценки [15]. В исследовании Monasta L c соавт. распространенность избыточной массы тела у девочек 5 лет составила 3.4\% по ВО3 и 15.3\% по критериям IOTF [16]. А в работе Salanave B с соавт. при обследовании 2525 детей распространенность избыточной массы тела у девочек 7-9 лет составила 25,2\% по ВО3 и 19,5\% по критериям IOTF, у мальчиков - 27,6\% и 17,4\% соответственно [17].

В нашей стране в настоящее время, согласно федеральным клиническим рекомендациям, с учетом рекомендаций BO3, ожирение у детей и подростков от 0 до 19 лет следует определять как ИМТ, равный или более +2,0 SDS ИMT, а избыточную массу тела от +1,0 до +2,0 SDS ИMT. Нормальная масса тела диагностируется при значениях ИМТ в пределах $\pm 1,0$ SDS ИMT. [18, 19].

Другие способы оценки ожирения у детей: окружность талии, биоимпеданс, толщина кожной складки, КТ, МРТ имеют скорее мотивационное или научное значение и в практическом здравоохранении не используются. Так, определение окружности талии у детей имеет скорее мотивационное (оценка в динамике), чем диагностическое или прогностическое значение. Данная величина значимо варьирует в зависимости от этнической принадлежности, возраста, пола и роста ребенка. Единые унифицированные критерии оценки окружности талии для детей в настоящее время отсутствуют. Ряд стран (США, Австалия, 
Канада, Англия, Болгария, Турция и др.) разработали свои национальные перцентильные таблицы величины окружности талии, наглядно демонстрирующие значительную вариабельность ее "нормальных" значений. Кроме того, у детей значение величины окружности талии как маркера висцерального ожирения не определено, а соотношение окружностей талии и бедер считается неинформативным.
Это связано как с изменяющимися пропорциями тела по мере роста ребенка, так и с малочисленностью работ по данной теме.

Таким образом, ведущим на сегодняшний день критерием диагноза ожирения у детей и подростков является определение перцентили или стандартного отклонения величины ИМТ с поправкой на возраст и пол ребенка.

\section{СПИСОК ЛИТЕРАТУРЫ | REFERENCES}

1. Keys A, Fidanza F, Karvonen MJ, Kimura N, Taylor HL. Indices of relative weight and obesity. J Chronic Dis. 1972;25(6-7):329-343. doi: 10.1016/0021-9681(72)90027-6

2. Cole TJ. A method for assessing age-standardized weight-for-height in children seen cross-sectionally. Ann Hum Biol. 1979;6(3):249-268. doi: 10.1080/030144679000007252

3. Rolland-Cachera MF, Deheeger M, Bellisle F, et al. Adiposity rebound in children: a simple indicator for predicting obesity. Am $J$ Clin Nutr. 1984;39(1):129-135. doi: 10.1093/ajen/39.1.129

4. Veldhuis JD, Roemmich JN, Richmond EJ, et al. Endocrine Control of Body Composition in Infancy, Childhood, and Puberty. Endocr Rev. 2005;26(1):114-146. doi: 10.1210/er.2003-0038

5. Must A, Dallal GE, Dietz WH. Reference data for obesity: 85 th and 95 th percentiles of body mass index (wt/ht2) and triceps skinfold thickness. Am J Clin Nutr. 1991;53(4):839-846. doi: 10.1093/ajen/53.4.839

6. Бахрах, И.И. Физическое развитие детей школьного возраста. В Сб. науч. тр.: Медицина подросток и спорт / Под ред. Бахраха И.И., Дорохова Р.Н. - Смоленск: СГИФК, 1975. - С.5-38. [Bakhrakh II. Fizicheskoe razvitie detei shkol'nogo vozrasta. In: Bakhrakh II, Dorokhov RN, editors. Meditsina podrostok i sport. Smolensk; 1975. p.5-38. (In Russ.)]

7. Воронцов И.М. Закономерности физического развития детей и методы его оценки: Учебно-методическое пособие. - Л.: ЛПМИ, 1986. - 55 c. [Vorontsov IM. Zakonomernosti fizicheskogo razvitiya detei i metody ego otsenki: Uchebno-metodicheskoe posobie. Leningrad: Leningrad Pediatric Medical Institute (LPMI); 1986. p. 55 (In Russ).]

8. Пропедевтика детских болезней. учебник для студентов мед. вузов. / Под ред. Мазурина А.В., Воронцова И.М. - СПб: Фолиант, 2001. 926 c. [Mazurin AV, Vorontsov IM, editors. Propedevtika detskikh boleznei. uchebnik dlya studentov med. vuzov. Saint Petersburg: Foliant; 2001. p. 926 (In Russ).]

9. Ogden CL, Kuczmarski RJ, Flegal KM, et al. Centers for Disease Control and Prevention 2000 Growth Charts for the United States: Improvements to the 1977 National Center for Health Statistics Version. Pediatrics. 2002;109(1):45-60. doi: 10.1542/peds.109.1.45

10. Centre for Public Health Excellence at NICE (UK); National Collaborating Centre for Primary Care (UK). Obesity: The Prevention, Identification, Assessment and Management of Overweight and Obesity in Adults and Children [Internet]. London: Nation- al Institute for Health and Clinical Excellence (UK); 2006 Dec (NICE Clinical Guidelines, No. 43.) [cited 2019 Apr 9] Available from: https://www.ncbi.nlm.nih.gov/books/NBK63696/

11. Cole TJ. Establishing a standard definition for child overweight and obesity worldwide: international survey. BMJ. 2000;320(7244):1240-1240. doi: 10.1136/bmj.320.7244.1240

12. de Onis M. Development of a WHO growth reference for school-aged children and adolescents. Bull World Health Organ. 2007;85(09):660-667. doi: 10.2471/BLT.07.043497

13. Карпушкина А.В., Геппе Н.А. Профилактика ожирения у детей в системе здравоохранения // Доктор.Ру. - 2015. - №13(144). C.8-11. [Karpushkina AV, Geppe NA. Prevention of Pediatric Obesity at Healthcare-System Level. Doktor.ru. 2015.13(114):8-11. (In Russ.)]

14. ELLIS KJ, SHYPAILO RJ, ABRAMS SA, WONG WW. The Reference Child and Adolescent Models of Body Composition: A Contemporary Comparisona. Ann N Y Acad Sci. 2006;904(1):374-382. doi: 10.1111/j.1749-6632.2000.tb06486.x

15. Rolland-Cashera MF. Childhood obesity: current definitions and recommendations for their use. Int J Pediatr Obes. 2011 Oct;6 (56):325-31. doi: 10.3109/17477166.2011.607458.

16. Monasta L, Lobstein T, Cole TJ, et al. Defining overweight and obesity in pre-school children: IOTF reference or WHO standard? Obes Rev. 2011;12(4):295-300. doi: 10.1111/j.1467-789X.2010.00748.x

17. Salanave B, Peneau S, Rolland-Cachera M-F, Hercberg S, Castetbon $\mathrm{K}$. Stabilization of overweight prevalence in French children between 2000 and 2007. Int J Pediatr Obes. 2009;4(2):66-72. doi: 10.1080/17477160902811207

18. Диагностика и лечение ожирения у детей и подростков. В кн.: Федеральные клинические рекомендации (протоколы) по ведению детей с эндокринными заболеваниями / Под ред. И.И. Дедова и В.А. Петерковой. - М.: Практика, 2014, - C.163-183. [Dedov II, Peterkova VA, editors. Diagnostika i lechenie ozhireniya u detei i podrostkov. In: Federal'nye klinicheskie rekomendatsii (protokoly) po vedeniyu detei s endokrinnymi zabolevaniyami. Moscow: Praktika; 2014. p.163-183. (In Russ).]

19. Петеркова В.А., Нагаева Е.В., Ширяева Т.Ю. Методические рекомендации: Оценка физического развития детей и подростков. М.: Российская ассоциация эндокринологов, 2017. - C.98. [Peterkova VA, Nagaeva EV, Shiryaeva TYu. Metodicheskie rekomendatsii: Otsenka fizicheskogo razvitiya detei i podrostkov. Moscow: Russian Association of Endocrinologists; 2017. p.98 (In Russ).]

\section{ИНФОРМАЦИЯ ОБ АВТОРАХ [AUTHORS INFO]}

*Васюкова Ольга Владимировна, к.м.н. [Olga V. Vasyukova, MD, PhD]; ORCID: https://orcid.org/0000-0002-9299-1053; eLibrary SPIN: 6432-3934; e-mail: o.vasyukova@mail.ru

\section{ЦИТИРОВАТЬ:}

Васюкова О.В. Ожирение у детей и подростков: критерии диагноза // Ожирение и метаболизм. - 2019. - Т.16. №1. - C. 70-73. doi: 10.14341/omet10170

\section{TO CITE THIS ARTICLE:}

Vasyukova OV. Obesity in children and adolescents: diagnosis criteria. Obesity and metabolism. 2019;16(1):70-73. doi: $10.14341 /$ omet 10170 